\title{
Genetic effects stack up in some people with autism
}

\author{
BY LAURA DATTARO
}

23 NOVEMBER 2021

\section{Listen to this story:}

https://www.spectrumnews.org/wp-content/uploads/2021/11/audio-c9327639-d9ed-424c-bfaea9655d95a4f9-encodings.mp3

Rare genetic variants that alter the expression of genes in the brain contribute to autism in people who also have a rare autism-linked mutation, according to a new study. The findings help explain how some 'second hits' drive autism in people with known mutations, the researchers say.

The study analyzed families carrying deletions of DNA in the chromosomal region 16p12.1. About 30 percent of people with this deletion are autistic; 90 percent inherit the mutation from a parent, who often has mild or no neuropsychiatric traits.

Children with autism and the $16 \mathrm{p}$ deletion who carry other genetic variants - often inherited from the parent without the deletion - tend to have more profound traits than those without second genetic 'hits,' the researchers showed in 2018.

Unpublished work from the same group supports those 'second hit' findings: In an analysis of 113 families with the deletion, carrier parents tended to have different and often milder neuropsychiatric traits than their carrier children. The researchers presented the work at the 2021 American Society of Human Genetics meeting last month.

The new work shows that the second hits in the children affect autism traits largely through altering the expression of other genes.

"We already suspect that these mechanisms are in play, but what this study does a nice job of showing is that it's the combination of these features," says Dan Arking, professor of genetic medicine at Johns Hopkins University in Baltimore, Maryland, who was not involved in the work. 


\section{Spectrum | Autism Research News}

https://www.spectrumnews.org

"That is nice to be able to demonstrate."

Together, the findings could also help clinicians better advise families about their children's likely developmental trajectories, says lead investigator Santhosh Girirajan, associate professor of genomics at Pennsylvania State University. Children with the deletion and a family history of psychiatric conditions may be more likely to have more profound traits.

"There could be a lot of things going on, but in general, family history plays a major role," Girirajan says.

\section{Differing expression:}

Girirajan and his colleagues sequenced the whole genomes of 32 people from five families. Each family includes some members who carry the deletion: 10 children -8 of them autistic -6 nonautistic parents and 3 non-autistic grandparents. They also collected blood cells to establish cell lines to measure gene expression, plus information about the participants' health and developmental traits.

More than 1,500 genes were expressed differently between carriers and non-carriers, the team found. And many of the genes are strongly linked to autism, including FOXP1, ANK3 and EP300.

The carrier children, though, shared an equal number of expression changes with their non-carrier parents - who likely passed on the additional genetic 'hits' - and their carrier parents, a result Girirajan calls "interesting."

The findings were published in Genome Medicine in October.

DNA sequencing revealed 25 groups of additional rare variants in the cohort, at least one of which occurred in about 10 percent of genes with expression changes.

Many of the variants affect genes belonging to a brain-specific gene-interaction network, which suggests they could influence gene-expression levels in the developing brains of carrier children, the researchers say.

In several instances, the combination of gene-expression differences from the 16p12.1 deletion and an additional hit was greater than the total effect from either mutation alone, the team found, suggesting an interplay between the two.

"The synergistic effect is 1 plus 2 is 5 ," Girirajan says.

\section{Genetic background:}




\section{Spectrum | Autism Research News}

https://www.spectrumnews.org

It's useful that the team attempted to go beyond associations to identify mechanisms by which additional genetic hits act, Arking says. Future work with more non-carrier siblings would make the findings more robust, as would analyzing gene expression in neurons generated from stem cells from the participants, rather than the blood cell lines used in this study.

But the work helps to demonstrate how the same deletion can lead to such different outcomes in people, says Nicola Grissom, assistant professor of psychology at the University of Minnesota in Minneapolis, who was not involved in the work.

"It's really important to embrace the fact that it's not deterministic, but to try to understand what this gene variant actually does and how it might do it," she says.

And it shows the importance of better understanding genetic background, not only in people but also in mouse models of autism-linked mutations, she says.

"That is a really important and fundamentally underexplored question," Grissom says.

Cite this article: https://doi.org/10.53053/FWIZ2655 